\title{
Optimization of cardiac resynchronization therapy based on speckle tracking
}

\author{
Sipula $\mathrm{D}^{1}$, Kozak $\mathrm{M}^{3}$, Sipula $\mathrm{J}^{1}$, Homza $\mathrm{M}^{1}$, Plasek $\mathrm{J}^{1}$, Furst $\mathrm{T}^{2}$ \\ Cardiovascular Department of the University Hospital in Ostrava, Ostrava, Czech Republic, \\ Faculty of Medicine, Masaryk University, Brno, Czech Republic. sipula@email.cz
}

\begin{abstract}
OBJECTIVES: The aim of this study was to evaluate the correlation between the change in heart strains and the success rate of Cardiac Resynchronization Therapy (CRT) optimization. We further explored the benefit of speckle tracking for CRT.

METHODS: In this prospective cohort study, CRT-Ds were implanted to 60 patients. 3 months later, the response was evaluated. In the non-responders, optimization based on speckle tracking was performed. The AV interval was optimized with respect to the quality of left ventricle filling and the VV interval was optimized with respect to heart strains. After a further three months, the optimization success was evaluated.

RESULTS: Thirty-nine patients responded well to the initial CRT. The response was independent of etiology; the subsequent optimization was however more successful in dilated cardiomyopathy (DCM) (8 out of 9) than in ischemic heart disease (IHD) patients (3 out of 10 responded). The ejection fraction increase and area strain were the best predictors of NYHA improvement.

CONCLUSION: AV and VV optimization in patients who do not respond well to initial CRT seems to have better results in patients suffering from DCM. Speckle tracking (specifically A-strain) may be used to guide CRT optimization (Tab. 2, Fig. 3, Ref. 22). Text in PDF www.elis.sk.

KEY WORDS: cardiac resynchronization therapy, heart failure, optimization, speckle tracking, strain.
\end{abstract}

\begin{abstract}
Abbreviations: CRT - cardiac resynchronization therapy, CRT-D - cardiac resynchronization therapy with defibrillator, NYHA New York Heart Association, DCM - dilatation cardiomyopathy, IHD - ischemic heart disease, ICD - implantable cardioverter defibrillator, LBBB - left bundle branch block, AV - atrioventricular, VV - interventricular, EF - ejection fraction, VTI - velocity time integral, SV - systolic volume
\end{abstract}

\section{Introduction}

Cardiac Resynchronization Therapy (CRT) is an efficient nonpharmacological treatment of chronic heart failure. If a patient is indicated to be suitable for CRT, the probability of a positive clinical outcome is high. Based on the PROSPECT, SMART-AV, and ECHO-CRT studies, the optimal CRT indication criteria seem to be the following: heart failure NYHA grade III-IV, QRS complex duration over $130 \mathrm{~ms}$, chronic left bundle branch block (LBBB)

${ }^{1}$ Cardiovascular Department of the University Hospital in Ostrava, Ostrava, Czech Republic, Faculty of Medicine, Masaryk University, Brno, Czech Republic, ${ }^{2}$ Department of mathematical analysis and applications of mathematics, Faculty of Science, Palacky University, Olomouc, Olomouc, Czech Republic, and ${ }^{3}$ Clinic of Internal Medicine - Cardiology, University Hospital Brno, Brno, Czech Republic

Address for correspondence: D. Sipula, MD, Cardiovascular department of the University Hospital in Ostrava, 17. listopadu 1790, CZ-708 00 Ostrava, Czech Republic. Phone: +420.604475694 and left ventricular ejection fraction below $35 \%$ (1-4). In patients with a sinus rhythm, a higher treatment success rate is reported than in patients with chronic atrial fibrillation (5).

CRT aims to improve the functional state of a patient with chronic heart failure. Success may be measured both subjectively on the NYHA scale and objectively by overall mortality. CRT works by reducing the dyssynchrony of the ventricular contraction (interventricular delay), which leads to an improved systolic function of the left ventricle. However, CRT does not improve the functional state of all patients. Cardiologists have long been trying to find methods to identify potential responders or to further optimize CRT to improve the condition of non-responders. These methods were traditionally based on the tracking of the left ventricle mechanical function by means of echocardiography. In the TM mode, the intraventricular delay was assessed between the peaks of the systolic activity of the septum and the back wall of the LV. It was also possible to track this quantity by observing myocardial movement using the tissue Doppler technique (6). In PW Doppler mode, the interventricular delay was assessed by observing the difference between the beginning of the systolic discharge of the left and right ventricles. According to various studies (1-4), these techniques had only limited informative value because they only tracked the velocity of the myocardial movement. After the PROSPECT study, echocardiograph manufacturers introduced software which made it possible to track and evaluate the myocardial deformation - so-called speckle tracking. 
Myocardial deformation is quantified by means of the socalled strains. Strain is defined as the change in length of a segment of myocardium relative to its resting length and is expressed as a percentage (7). Global strain is defined as the sum of all the strains of all left ventricle segments. Echocardiography provides 4 types of strains: longitudinal strain ( $\mathrm{L}$, measures myocardial deformation in the longitudinal axis), circumferential strain (C, measures the deformation in the short axis), radial strain ( $R$, measures the thickening of myocardial walls during systole), and area strain (A, a combination of the longitudinal and circumferential strains). $\mathrm{L}, \mathrm{C}$, and A strains are normally negative because they express myocardial contraction, the R strain is usually positive because it captures myocardial dilation. Strain evaluation in $2 \mathrm{D}$ did not bring the expected success because the simplified 2D projection was too crude. Only with 3D echocardiography and the full 3D evaluation can better results be expected (8).

According to a study by Marek et al., it is possible that some selected subgroups, for example non-responders, might benefit from the strategy of AV and VV optimization; there is however not enough evidence that it is beneficial to all CRT patients (1). Some studies have indicated that atrioventricular and interventricular suboptimal delays are predictors of poor CRT, and highlighted the significant clinical benefit after atrioventricular and interventricular optimization $(9,10)$.

Compared with conventional ECG optimization, optimization with Doppler and 3D echocardiography protocol resulted in a significantly higher response rate, improved LV systolic function, and may be used to select the optimal AV and VV intervals in CRT (11). The ability of speckle tracking to predict CRT response in patients has also been demonstrated in multiple recent studies (12-16). All the above suggests that speckle tracking is a promising method, which has, however, not been utilized to the fullest so far - it has been used to identify patients' response to CRT but not used as a tool for optimizing CRT performance.

In this work, we took things a step further, evaluating all four strains (L, C, R, and A). The aim of this study is to assess the relation of the strain values to the CRT response, both by means of the subjective NYHA score and by means of echocardiographic parameters such as the ejection fraction, aortic valve blood flow (measured by VTI), and the systolic volume. Moreover, we wanted to explore the possibility of using the strain values for the atrioventricular and interventricular optimization of biventricular stimulation in those patients who did not show significant improvement after the initial CRT implant.

\section{Methods}

\section{Study group}

This was a prospective cohort study. All patients who were admitted over to the cardiovascular center of the Faculty hospital in Ostrava for heart failure, and met the following criteria for ICD implantation, were included: symptomatic heart failure with NYHA III/IV, pharmacological treatment options exhausted, left ventricle ejection fraction below $30 \%$, and QRS duration over

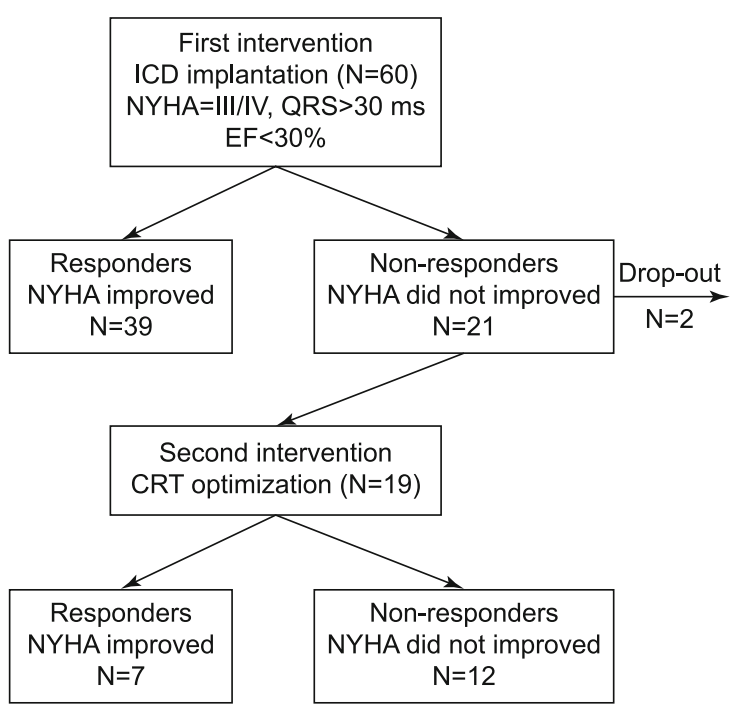

Fig. 1. Time-course of the study.

130 ms. The study was approved by the local Ethics Committee. All patients signed an informed consent.

\section{Medical examination and therapy}

All patients enrolled into the study underwent a basic physical examination, ECG, and echocardiographic examination including 3D echocardiography. Usual parameters were evaluated, such as the dimensions of all the heart segments, blood flow through valves, and the kinetics of the right and left ventricles. In the subsequent analyses, we worked especially with the left ventricle ejection fraction, time-velocity integral (TVI) of the blood flow through the aortic valve, systolic left ventricle volume, and global L, C, and R strains.

After the initial examination, a biventricular ICD was implanted in all of the patients according to the standard procedure guidelines (17). An electrode was placed into the right atrium (unless they presented with permanent atrial fibrillation), right ventricle, and into the lateral branch of the coronary sinus. After the implantation, the defibrillator was set for the maximal stimulation of the ventricles with the VV interval set to decrease the duration of the QRS complex as much as possible. All the patients were invited for a check-up in three months.

After these three months, based on the results of the ICD implantation, patients were divided into responders and non-responders (Fig. 1). Responders were defined as those patients who reported NYHA improvement of at least one category. All the nonresponders underwent (on an out-patient basis) atrioventricular optimization by CRT, together with a complete echocardiographic examination including 3D echocardiography.

The optimization proceeded as follows. First, the atrioventricular interval was optimized according to the blood flow through the mitral valve in order to ensure the best possible filling of the left ventricle. We observed the $\mathrm{E}$ and $\mathrm{A}$ waves and adjusted the $\mathrm{AV}$ interval so that the waves did not overlap and one followed 
Tab. 1. Description of the myocardial characteristics before and after the first and the second intervention. Left ventricular ejection fraction (EF), longitudinal strain (L), circumferential strain (C), area strain $(A)$, radial strain $(R)$, the difference in volume-time integral through the aortal valve (VTI_diff), and the difference in systolic left ventricle volume (SV_diff).

\begin{tabular}{lccccccc}
\hline & \multicolumn{2}{c}{ Initial ICD implantation: $\mathrm{n}=60$} & & \multicolumn{3}{c}{ CRT optimization: $\mathrm{n}=19$} \\
\cline { 2 - 3 } & Median & Min & Max & & Median & Min & Max \\
\hline age & 69 & 31 & 85 & & 69 & 31 & 85 \\
EF_before & 25 & 15 & 35 & & 25 & 12 & 32 \\
EF_after & 33 & 15 & 56 & & 29 & 15 & 40 \\
L_before & 7 & 1 & 14 & & 7 & 3 & 12 \\
L_after & 11 & 2 & 18 & & 8 & 4 & 15 \\
C_before & 6 & 1 & 20 & & 7 & 2 & 14 \\
C_after & 11 & 1 & 22 & & 10 & 4 & 16 \\
A_before & 10 & 1 & 27 & & 11 & 3 & 18 \\
A_after & 18 & 1 & 33 & & 14 & 3 & 24 \\
R_before & 16 & 1 & 44 & & 17 & 3 & 28 \\
R_after & 27 & 2 & 78 & & 25 & 2 & 48 \\
VTI_diff & 0.30 & -0.40 & 1.35 & & 0.18 & -0.14 & 0.56 \\
SV_diff & 0.33 & -0.46 & 1.90 & & 0.14 & -0.16 & 1.76 \\
\hline
\end{tabular}

the other smoothly in order to avoid both truncation and fusion. Next, the interventricular interval was optimized according to the strains. We adjusted the VV interval to reach the optimal value of all three strains. For the $\mathrm{C}$ and $\mathrm{L}$ strains, the maximum negative value was targeted, while in the case of $\mathrm{R}$ strain, the maximum possible positive value was sought.

After a further three months, all the patients who underwent this optimization were again invited for an examination. This examination was performed by three different experienced echocardiographers. The initial setting and the optimization of the ICD were always performed by the same arrhythmologist/electrophysiologist.

\section{Statistical analysis}

Several statistical tests were used to compare the difference in parameters and/or outcomes between the group of responders and non-responders. When testing for a difference in a continuous variable (e.g. age), the Kruskal-Wallis test was used. For discrete parameters (e.g. sex), chi square asymptotic test in a contingency table was used. If the frequencies in the table were too small, Fisher exact factorial test was used (Tab. 1). All tests were performed at 0.05 level of significance. Data processing, visualization, and statistical testing were performed in MATLAB and Statistics Toolbox Release 2012b, The MathWorks, Inc., Natick, Massachusetts, United States.

\section{Results}

The cohort consisted of 49 men and 11 women. Median age at diagnosis was 68.5 years, age ranged between 31 and 85 years. In 31 patients, the heart failure was of an ischemic etiology (or coronary artery disease, CAD) while 29 patients suffered from dilatation cardiomyopathy (DCM). 45 patients exhibited a sinus rhythm while atrial fibrillation was observed in 15 patients.
Tab. 2. Change in NYHA with both interventions. Rows: NYHA before the intervention, columns: NYHA after the intervention.

\begin{tabular}{lccccccccc}
\hline & \multicolumn{3}{c}{ Initial ICD implantation } & \multicolumn{5}{c}{ CRT optimization } \\
\hline NYHA & 1 & 2 & 3 & 4 & NYHA & 1 & 2 & 3 & 4 \\
\hline 3 & 6 & 32 & 19 & 2 & 3 & 1 & 6 & 11 & 0 \\
4 & 0 & 1 & 0 & 0 & 4 & 0 & 0 & 0 & 1 \\
\hline
\end{tabular}

Table 2 shows the NYHA stratification of the patients before and after both interventions. After the first intervention, there were 21 non-responders and 39 responders. The second intervention was performed in 19 patients (one refused and the other one was lost from follow-up). Only one of the patients whose NYHA increased after the first intervention underwent the optimization procedure. Responders were further cardiologically examined on an outpatient basis. Out of the 18 non-responders who underwent the optimization, 7 responded well and 12 remained non-responsive.

In the first intervention (CRT-D implantation), the response was independent of the sex; however, the responders were significantly older $(p=0.02)$ (Fig. 2). Median responder age was 70 while for non-responders, the median age was 67 . A positive response to the first intervention was associated neither with the etiology of the heart failure nor with heart rhythm. The rhythm was not associated with age; however, the patients suffering from IMP were naturally older (median age 71 years) than patients with DMP (median age 61 years). Thus, this effect may confound the age-dependence of the response.

Further, we wanted to know which of the echocardiographic parameters (EF, TVI, SV, and all the strains) were the best predictor of NYHA improvement after the first intervention. We found that the ejection fraction is a very strong predictor of NYHA improvement. Patients whose NYHA did not decrease after the first intervention exhibited an EF increase of at most $0-5$ percentage points. Patients whose NYHA decreased by one category showed an EF increase of around 15 percentage points, and patients whose NYHA decreased by two categories showed an EF increase of around 25 percentage points. All three groups (no NYHA improvement, NYHA+1, and NYHA+2) were significantly different: the Kruskal-Wallis test rejects the hypothesis of the three groups being drawn from the same distribution at $\mathrm{p}=3 \mathrm{e}-9$. Among the strains, we found the area strain to be the best predictor of NYHA improvement. Although it clearly separates the NYHA+0 and NYHA+1 groups, the overlap between $\mathrm{NYHA}+1$ and NYHA+2 groups is larger than in the case of EF. Nevertheless, the increase in the A strain is significantly associated with NYHA improvement, $\mathrm{p}=5 \mathrm{e}-$ 8. In fact, all of the potential predictors (EF, TVI, SV, and all the strains) clearly discriminate responders from non-responders. EF and the A-strain seem to have the strongest association to NYHA and least overlap among the three NYHA improvement groups.

Among the 19 optimized patients, 9 suffered from DCM and 10 from CAD. After the optimization, 8 out of 9 DCM patients responded but only 3 out of $10 \mathrm{CAD}$ responded (Fig. 3 ). This effect is statistically significant $(p=0.02)$ despite the small sample size. A borderline association with age was found after the second intervention ( $p=0.07)$. The median age of the responders was 48 

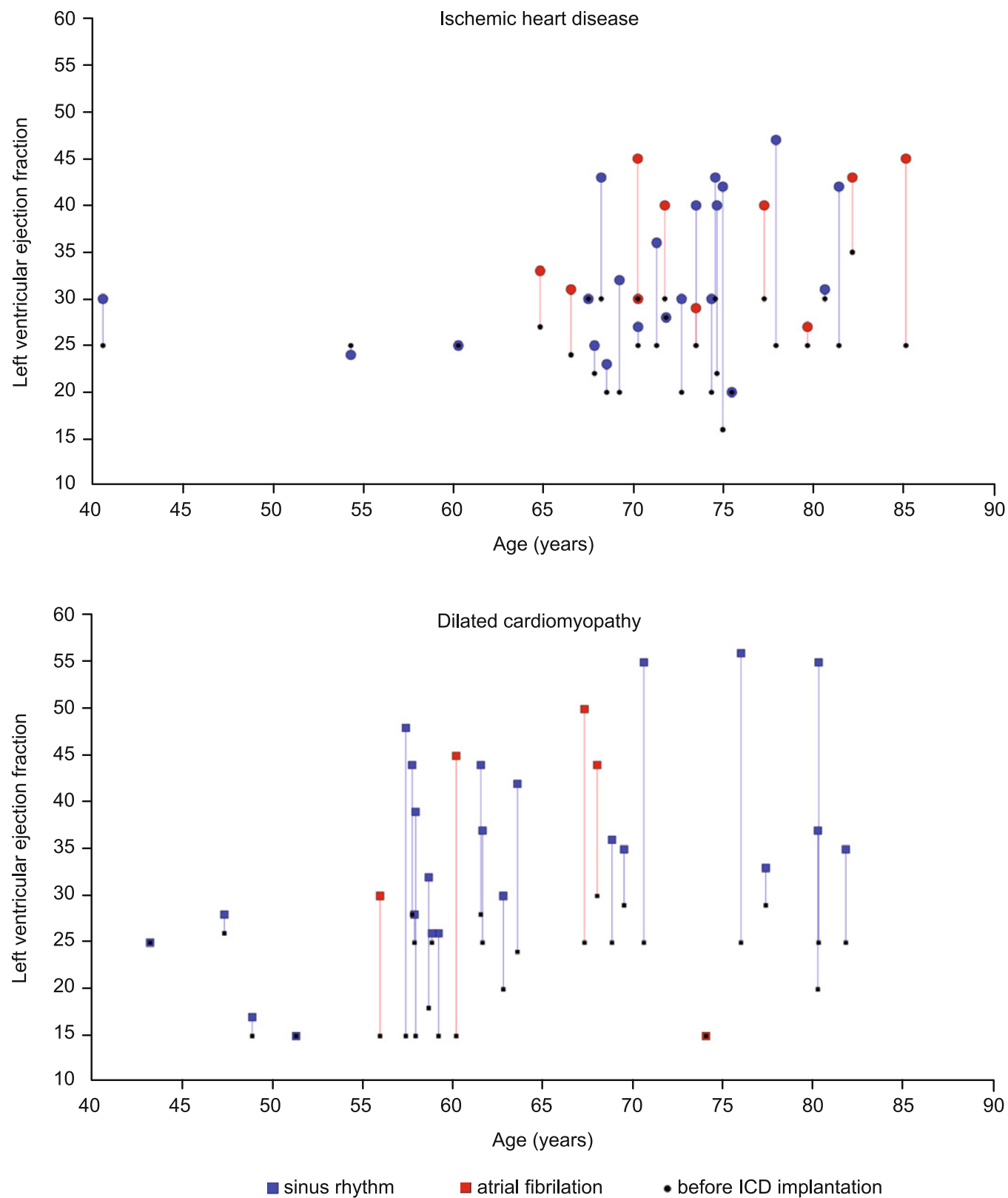

Fig. 2. Visualization of the patients' status change after the first intervention (ICD implantation). Age and the left ventricular ejection fraction are plotted against each other. Each vertical line connects the state of the respective patient before the intervention (a black square) and after the intervention. The upper panel shows patients suffering from CAD, and the lower patients suffering from DCM (both graphs have the same scale). The type of rhythm is color coded. It is clearly visible that CAD patients are generally older. DMC patients exhibit a larger increase in EF than IMC patients. In both groups, interventions with problematic outcomes appear to occur in younger patients.

years while the median age of non-responders was 69 years. The entire optimized group contained three patients with atrial fibrillation, none of whom responded to the second intervention.

\section{Discussion}

In our prospective cohort study, we monitored parameters before and after resynchronization therapy and tried to optimize biventricular devices to reach as many responders as possible. In total, we achieved $65 \%$ of responders in 60 patients. This correlates with large prospective trials with $69 \%$ of responders in the PROSPECT trial (18), $66 \%$ in the MIRACLE trial (19) and 64 $\%$ in the CARE-HF trial (20). Patients were indicated for resynchronization therapy according to valid guidelines, and the initial patient data were similar to a much larger, prospective randomized PROSPECT (18) study involving 498 patients. In compari- 


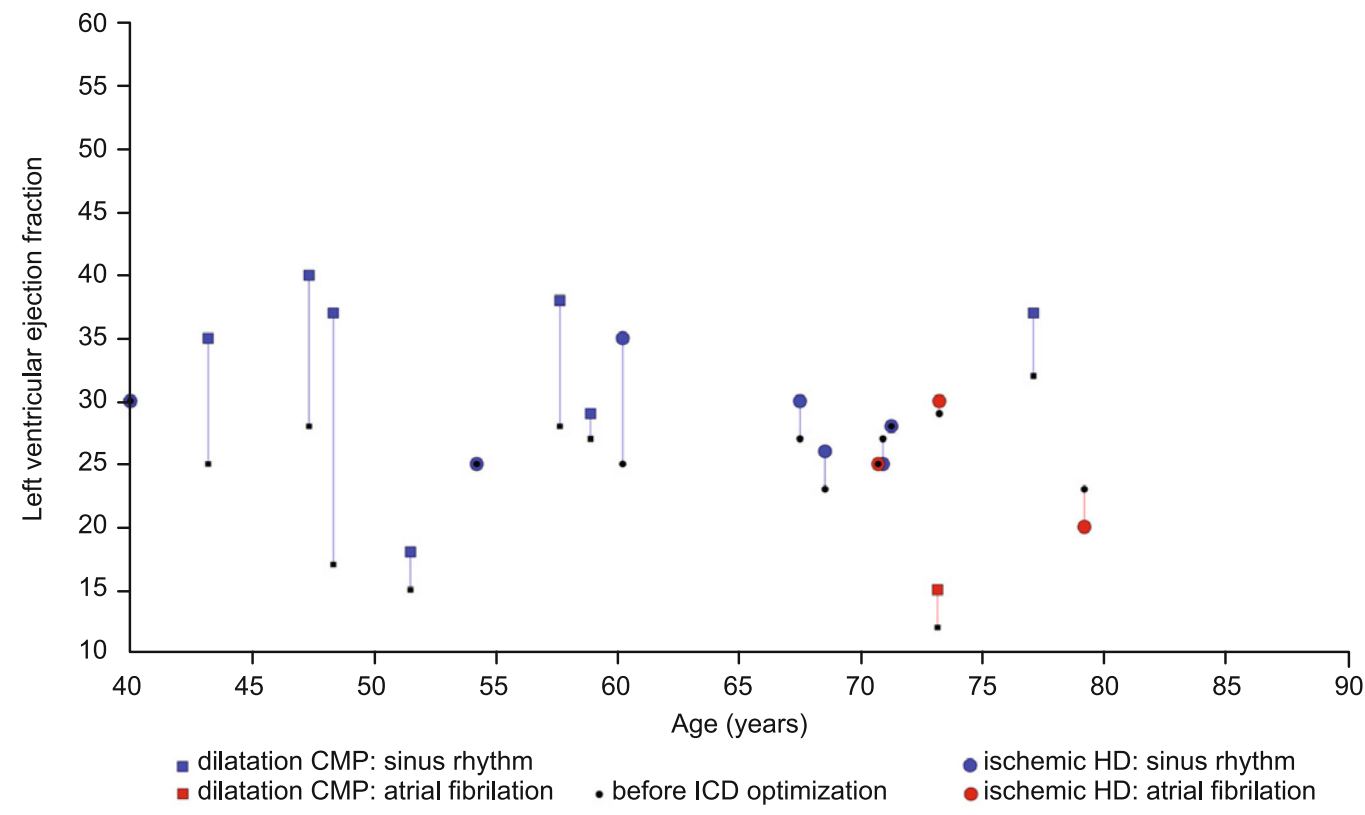

Fig. 3. Visualization of the patients' status change after the second intervention (CRT optimization). Age and the left ventricular ejection fraction are plotted against each other. Each vertical line connects the state of the respective patient before the intervention (a black square) and after the intervention. Different symbols code the etiology: the type of rhythm is color coded. It is clearly visible that DMC patients benefit more from the second intervention with the largest EF increase occurring for younger patients.

son, the average initial EF LK was identical at $25 \%$, the number of patients with CAD $54 \%$ in PROSPECT vs $52 \%$ in our study, and the male representation was $71 \%$ vs $81 \%$.

Patients in the DCM group responded better to CRT optimization than patients suffering from CAD. This is actually in accordance with the expectations as in cases of CAD, an irreversible myocardial scar may already have formed, preventing the function of the CRT device. In our report, the number of CAD responders was $48 \%$, while the DCM group responded in $82 \%$. These findings correlate with other randomized trials, such as CARE-HF and PROSPECT, where the number of CAD vs. DCM responders was $54 \%$ vs $79 \%$ and $64 \%$ vs $75 \%$ respectively $(18,20)$.

Although not statistically significant at the level of $\mathrm{p}<0.05$, a borderline significance was detected for the age variable $(\mathrm{p}=$ 0.07 ). We however believe that in this case, etiology is the true predictor of the response as the better responding DCM patients are significantly younger than CAD patients. Among all the potential predictors of response (EF, TVI, SV, and all the strains), $\mathrm{EF}$ and the A-strain again exhibited the highest discrimination of responders and non-responders. Despite the small sample, any one of the potential predictors was able to discriminate responders from non-responders with statistical significance.

In this small study, we have shown that strains provide very similar information to EF, so it can only be assumed that CRTD devices can be optimized by means of strains with good results. The most appropriate strain for CRT optimization seems to be the area strain which is a combination of the longitudinal and circumferential strains. The area strain predicts NYHA improvement as well as the EF. Therefore, we think it is reasonable to use the area strain together with EF for the optimization of CRT-D.

According to the current 2016 guidelines for heart failure therapy (17), biventricular device optimization of AV and VV intervals is not recommended for all patients. Our study advocates for such optimization, especially in patients with sinus rhythm suffering from DCM who did not respond well to the first intervention. In a study by Mullens et al. concerning CRT non-responders, inadequate AV setting was identified as the cause of non-response in up to $47 \%$ of patients. Setting adjustment was associated with long-term positive clinical outcomes (21).

This study does not provide evidence as to whether it is better to optimize the CRT-D based on the strain measurements or on the EF. A double-blinded placebo-controlled randomized study with a significantly higher number of patients would be needed to bring such evidence; the aim of this paper is to indicate that the use of speckle tracking for optimizing CRT-D performance in non-responders might be a promising technique worthy of further research. Good and consistent echocardiographic imaging is a prerequisite for the correct assessment of the strains. Thus, an experienced echocardiographer is needed. Optimization of CRT by means of strains is rather time-consuming and it requires the simultaneous presence of an experienced echocardiographer, an arrhythmologist, and the appropriate technical background (22). 


\section{Conclusion}

In our small single-center study, we implanted a biventricular CRT-D and tuned it in order to reach the minimum QRS complex duration. Subsequently, we optimized the CRT in non-responders using 3D echocardiography speckle tracking to achieve the most efficient AV and VV intervals. The results show that it is beneficial to perform such optimization in patients who do not respond to CRT in the first place. Patients suffering from dilatation cardiomyopathy respond especially well to the optimization. Out of 9 DCM patients indicated for the optimization, 8 improved by at least one category in the NYHA scale. On the other hand, out of 10 patients suffering from ischemic cardiomyopathy, only three responded well to the optimization.

Our results show that it is possible to use strains for CRT optimization. The area strain in particular seems to predict NYHA improvement very well. The area strain also correlates very well with the left ventricle ejection fraction.

\section{References}

1. Marek J et al. Echocardiography and cardiac resynchronization therapy. Cor et Vasa 2016; 58 (3): e340-e351.

2. Bax JJ et al. Left ventricular dyssynchrony predicts benefit of cardiac resynchronization therapy in patients with end-stage heart failure before pacemaker implantation. Am J Cardiol 2003; 92 (10): 1238-1240.

3. Bax JJ, Gorcsan J 3rd. Echocardiography and noninvasive imaging in cardiac resynchronization therapy: results of the PROSPECT (Predictors of Response to Cardiac Resynchronization Therapy) study in perspective. J Am Coll Cardiol 2009; 53 (21): 1933-1943.

4. Ellenbogen KA et al. Primary results from the SmartDelay determined AV optimization: a comparison to other AV delay methods used in cardiac resynchronization therapy (SMART-AV) trial: a randomized trial comparing empirical, echocardiography-guided, and algorithmic atrioventricular delay programming in cardiac resynchronization therapy. Circulation 2010; 122 (25): 2660-2668.

5. Waggoner AD, de las Fuentes L, Davila-Roman VG. Doppler echocardiographic methods for optimization of the atrioventricular delay during cardiac resynchronization therapy. Echocardiography (Mount Kisco, N.Y.) 2008; 25 (9): 1047-1055.

6. Sakamaki $\mathbf{F}$ et al. Novel dyssynchrony evaluation by M-mode imaging in left bundle branch block. and the application to predict responses for cardiac resynchronization therapy. J Cardiol 2014; 64 (3): 199-206.

7. Shah AM, Solomon SD. Myocardial deformation imaging: current status and future directions. Circulation 2012; 125 (2): e244-248.

8. Wisotzkey BL, Soriano BD, Buddhe S. Comparison of two-dimensional and three-dimensional echocardiographic strain in children with CHD. Cardiol Young 2017; 27 (8): 1557-1565.
9. Mele D et al. Echocardiographic evaluation of cardiac dyssynchrony: Does it still matter? Echocardiography 2018; 35 (5): 707-715.

10. Galli E, Leclercq C, Donal E. Mechanical dyssynchrony in heart failure: Still a valid concept for optimizing treatment? Arch Cardiovasc Dis 2017; 110 (1): 60-68.

11. Sonne $\mathbf{C}$ et al. Three-dimensional echocardiographic optimization improves outcome in cardiac resynchronization therapy compared to ECG optimization: a randomized comparison. Pacing Clin Electrophysiol 2014; 37 (3): 312-320.

12. Poulidakis E et al. Echocardiography for prediction of 6-month and late response to cardiac resynchronization therapy: implementation of stress echocardiography and comparative assessment along with widely used dyssynchrony indices. Int J Cardiovasc Imaging 2019; 35 (2): 285-294.

13. Zhu M et al. The value of left ventricular strain-volume loops in predicting response to cardiac resynchronization therapy. Cardiovasc Ultrasound 2019; 17 (1): 3.

14. Fulati $Z$ et al. Speckle tracking echocardiography analyses of myocardial contraction efficiency predict response for cardiac resynchronization therapy. Cardiovasc Ultrasound 2018; 16 (1): 30.

15. Zweerink A et al. Strain imaging to predict response to cardiac resynchronization therapy: a systematic comparison of strain parameters using multiple imaging techniques. ESC Heart Fail 2018; 5 (6): 1130-1140.

16. Khidir MJH et al. Prognostic value of global longitudinal strain in heart failure patients treated with cardiac resynchronization therapy. Heart Rhythm 2018; 15 (10): 1533-1539.

17. Ponikowski P et al. 2016 ESC Guidelines for the diagnosis and treatment of acute and chronic heart failure: The Task Force for the diagnosis and treatment of acute and chronic heart failure of the European Society of Cardiology (ESC)Developed with the special contribution of the Heart Failure Association (HFA) of the ESC. Eur Heart J 2016; 37 (27): 2129-2200.

18. Chung ES et al. Results of the Predictors of Response to CRT (PROSPECT) trial. Circulation 2008; 117 (20): 2608-2616.

19. Abraham WT et al. Effects of cardiac resynchronization on disease progression in patients with left ventricular systolic dysfunction, an indication for an implantable cardioverter-defibrillator, and mildly symptomatic chronic heart failure. Circulation 2004; 110 (18): 2864-2868.

20. Cleland JG et al. The effect of cardiac resynchronization on morbidity and mortality in heart failure. N Engl J Med 2005; 352 (15): 1539-1549.

21. Mullens W et al. Insights from a cardiac resynchronization optimization clinic as part of a heart failure disease management program. J Am Coll Cardiol 2009; 53 (9): 765-773.

22. Sohaib SM et al. Cardiac resynchronisation therapy optimisation strategies: systematic classification, detailed analysis, minimum standards and a roadmap for development and testing. Int J Cardiol 2013; 170 (2): 118-131.

Received April 10, 2019. Accepted May 24, 2019. 